\title{
Effects of Grape Seed Extract, Vitamin C, and Vitamin E on Ethanol- and Aspirin-Induced Ulcers
}

\author{
Vivian Molina Cuevas, Yazmín Ravelo Calzado, Yohani Pérez Guerra, Ambar Oyarzábal Yera, \\ Sonia Jiménez Despaigne, Rosa Mas Ferreiro, and Daisy Carbajal Quintana
}

\author{
Pharmacology Department, Centre of Natural Products, National Centre for Scientific Research, \\ Avenue 25 and 158 Street Cubanacán, Havana City 6880, Cuba \\ Correspondence should be addressed to Vivian Molina Cuevas, vivian.molina@cnic.edu.cu \\ Received 9 August 2011; Revised 23 September 2011; Accepted 23 September 2011 \\ Academic Editor: Mustafa F. Lokhandwala
}

Copyright ( $\odot 2011$ Vivian Molina Cuevas et al. This is an open access article distributed under the Creative Commons Attribution License, which permits unrestricted use, distribution, and reproduction in any medium, provided the original work is properly cited.

\begin{abstract}
Effects of GSE and vitamins C and E on aspirin- and ethanol-induced gastric ulcer and associated increases of lipid peroxidation in rats were compared. Two experiments were conducted. Rats were randomized into eight groups: a negative control and seven groups that received aspirin or ethanol for ulcer induction: one positive control (vehicle) and six with VC, VE, or GSE (25 and $250 \mathrm{mg} / \mathrm{kg}$ ). Ulcer indexes and gastric levels of malondialdehyde (MDA) were quantified. VC, VE, and GSE (25 and $250 \mathrm{mg} / \mathrm{kg}$ ) decreased aspirin, and ethanol-induced ulcers and MDA values compared with positive control group. The magnitude of aspirin ulcer reduction was comparable for all treatments, and MDA decrease with GSE was higher than with VC and tended to be greater, albeit none significantly, than with VE. GSE was more effective than VC and VE for lowering the ethanol ulcers, while the decrease of MDA levels with GSE was greater than with VC, but comparable to that achieved with VE. GSE protected against ethanolinduced gastric ulcers more effectively than VC or VE, while its protection against aspirin ulcers was comparable for all treatments. GSE produced the greatest reductions of gastric MDA in both models.
\end{abstract}

\section{Introduction}

Peptic ulcer, a common gastrointestinal pathological condition, is due to the loss of the balance between aggressive and defensive factors of the gastric and duodenal mucosa. Aggressive factors against gastric mucosa include acid, pepsin, Helicobacter pylori, nonsteroidal anti-inflammatory drugs (NSAIDs), and ethanol, while local mucosal defensive factors include bicarbonate, mucus secretion, blood flow, cellular regeneration, and endogenous protective agents like prostaglandins (PG) and epidermal growth factors [1-3]. Also, increased oxidative stress is believed to be linked to the aggressive factors-induced gastric mucosal damage $[4,5]$.

The pathogenesis of NSAIDs-induced gastric ulceration includes the block of cyclooxygenase (COX) activity that leads to lower mucus and bicarbonate secretion, decreased mucosal blood flow, neutrophil infiltration, alteration of microvascular structures, and increase of acid and pepsinogen secretion. In addition, increased production of reactive oxygen species (ROS), increased lipid peroxidation, and neutrophil infiltration have demonstrated to play a role in the pathogenesis of NSAIDs-induced ulcers, including the aspirin-induced ulcer [6-11].

On the other hand, increased oxidative stress plays a key role in the pathogenesis of ethanol-induced gastric damage, so that as oral ethanol produces gastric mucosal lesions and erosions, it increases lipid peroxidation, raises hydroxyl radicals generation, and causes DNA damage, while it lowers the gastric content of reduced glutathione. Also, ethanolinduced ulceration is linked to reduced mucosa microcirculation and to increased apoptosis [12-16].

Vitamin C (VC) (ascorbic acid) is a water-soluble antioxidant that directly scavenges ROS, like superoxide and hydroxyl radicals, hydrogen peroxide, singlet oxygen, and hypochlorous acid, and also guarantees the chain-breaking antioxidant action of vitamin $\mathrm{E}$ (VE) by reducing the VE radical to VE $[17,18]$. In addition, $\mathrm{VC}$ has been shown to attenuate aspirin-induced gastric damage and to offer effective gastroprotection $[19,20]$. 
In turn, VE (alpha-tocopherol), a lipid-soluble antioxidant that scavenges ROS and functions as a chain-breaking antioxidant for peroxidation of membrane lipids, has been shown to inhibit neutrophils adhesion to endothelial cells and the oxygen production in activated neutrophils [2123]. Antiulcer effects of VE on aspirin- and ethanol-induced ulcers have been reported [24-26].

Grape seed extracts (GSEs), rich in flavonoids, mainly proanthocyanidin, have been shown to produce effective antioxidant effects [22-30]. Experimental studies have shown that oral administration of GSE lowers ROS generation and plasma protein carbonyl groups, while it enhanced the activity of the endogenous antioxidant system [27-30]. Clinical trials have confirmed the antioxidant effects of GSE [31-33]. The antiulcer activity of GSE has been also referred [33].

In light of these issues and to our knowledge (Entrez PubMed review up to July 2011), no previous study compared the preventive effects of GSE, VC, and VE against aspirin- and ethanol-induced gastric ulceration and associated increases of lipid peroxidation; this study was aimed to do such comparison.

\section{Materials and Methods}

2.1. Animals. Male Sprague Dawley rats (200-250 g) from the National Center for Laboratory Animal Production (CENPALAB, Havana City, Cuba) were adapted to laboratory conditions $\left(25 \pm 2{ }^{\circ} \mathrm{C}\right.$ of temperature, $60 \pm 5 \%$ of relative humidity, and 12-hour light/dark cycles) for 7 days. Food (rodent pellets from CENPALAB) and tap water were provided ad libitum.

Experiments were conducted in accordance to the Cuban guidelines of Animal Handling and the Cuban Code of Good Laboratory Practices (GLP), which follow international guidelines for the use and care of laboratory animals. Study protocol and animal use were approved, prior to study beginning, by an independent Animal Ethics Committee.

2.2. Administration and Dosage. GSE ( $85 \%$ in proanthocyanidine) came from Blackmores (Sydney, Australia), and VC (Cuban Pharmaceutical Industry) was prepared as a suspension in acacia gum/water vehicle (1\%), while VE (Carlson health, VIC, Australia), was suspended in a Tween $65 /$ water suspension (2\%). All suspensions were prepared daily, 1 hour before use and administered by oral gastric gavage ( $5 \mathrm{~mL} / \mathrm{kg}$ of body weight) for 10 days.

Two independent experiments were conducted, in which rats were randomized into eight groups: a negative control and seven groups that received aspirin or ethanol for ulcer induction: one orally treated with the vehicle (positive control) and the other six with 25 and $250 \mathrm{mg} / \mathrm{kg}$ of VC, VE, or GSE, respectively.

2.3. Experimental Induction of Gastric Ulcers. The animals were fasted for 24 hours before the experiments with free access to water.
In both experiments, rats were sacrificed in ether atmosphere and their stomachs were immediately removed for quantifying the lesions.

2.3.1. Aspirin-Induced Gastric Ulcers. Following one hour of administering the last doses of vehicle, GSE, VC, or VE, a single oral dose of aspirin $(300 \mathrm{mg} / \mathrm{kg}$ ) was given by gastric gavage. Five hours later, rats were sacrificed.

2.3.2. Ethanol-Induced Gastric Ulcers. We followed the procedure in accordance to Zengil et al. (1987) [34]. In brief, one hour after the last administering of the vehicle, GSE, $\mathrm{VC}$, or VE, ethanol (60\%) (1 mL/200 g body weight) was intragastrically administered to each rat. One hour later, rats were sacrificed.

2.4. Evaluation of Gastric Mucosal Damage. The stomachs were opened along the greater curvature and washed with saline solution. The lesions in the gastric mucosa were examined macroscopically using magnification 3x. Ulcer indexes were determined as the sum of the lengths of the whole gastric lesions (in $\mathrm{mm}$ ). Two independent, blinded observers performed the observations and measurements of lesion lengths [35].

2.5. Determination of Lipid Peroxidation (LP) in Gastric Mucosa. Aliquots of gastric mucosa were obtained by gentle scraped with a scalp. Lipid peroxidation was assessed as per the content of thiobarbituric reactive substances (TBARS) in the gastric mucosa and quantified in accordance to Ohkawa et al. 1979 method [36], which has been widely used for this propose [37-40]. Results were expressed as nmol of malondialdehyde (MDA)/mg of protein. The protein concentration was determined according to modified Lowry method [41].

2.6. Statistical Analysis. Comparisons among groups were performed with the Kruskal Wallis test, and paired comparisons with the Mann-Whitney $U$ test. The level of statistical significance was set at $\alpha=0.05$. All analyses were performed using Statistics software for Windows (Release 6.0, StatSoft; Inc, USA).

\section{Results and Discussion}

Oral administration of GSE, VC, and VE, all at 25 and $250 \mathrm{mg} / \mathrm{kg}$, prevented aspirin- and ethanol-induced gastric mucosal ulceration and reduced the increase of gastric MDA elicited by these aggressive agents. To our knowledge (Entrez PubMed review up to July 2011), this study is the first comparative study of the gastroprotective effect of GSE with those of VC and VE against aspirin- and ethanol-induced ulceration and concomitant increase of lipid peroxidation on the rat gastric mucosa.

No negative, but all positive controls exhibited typical aspirin-induced gastric ulcers. Aspirin increased significantly MDA gastric concentrations as compared to the negative controls (Table 1). Both doses of each treatment reduced 
TABLE 1: Effects of GSE, VC, and VE on ulcer indexes and MDA gastric concentrations in rats with aspirin-induced ulcer.

\begin{tabular}{|c|c|c|c|c|c|}
\hline Treatment & Doses $(\mathrm{mg} / \mathrm{kg})$ & Ulcer index $(\mathrm{mm})$ & I $(\%)$ & MDA (nmol/mg of pt) & I $(\%)$ \\
\hline Negative control (vehicle) & 0 & $0 \pm 0^{* * * *}$ & - & $1.02 \pm 0.21^{* *}$ & - \\
\hline Positive control (vehicle + aspirin) & 0 & $27.06 \pm 5.60$ & - & $12.28 \pm 0.81$ & - \\
\hline $\mathrm{VC}+$ aspirin & 25 & $10.91 \pm 2.33^{* *}$ & 59.7 & $8.7 \pm 0.28^{* * *}$ & 31.8 \\
\hline $\mathrm{VC}+$ aspirin & 250 & $8.36 \pm 1.23^{* * *}$ & 69.1 & $4.69 \pm 0.92^{* *}$ & 67.4 \\
\hline $\mathrm{VE}+$ aspirin & 25 & $13.72 \pm 3.60^{*}$ & 49.3 & $6.33 \pm 0.86^{* * *}$ & 52.8 \\
\hline $\mathrm{VE}+$ aspirin & 250 & $10.31 \pm 2.70^{*}$ & 61.9 & $3.11 \pm 0.43^{* * *}$ & 81.4 \\
\hline GSE + aspirin & 25 & $11.56 \pm 3.50^{*}$ & 57.3 & $5.11 \pm 0.79 * * * \mathrm{a}$ & 63.6 \\
\hline GSE + aspirin & 250 & $11.81 \pm 3.03^{*}$ & 56.3 & $1.95 \pm 0.28^{* * * a t}$ & 91.7 \\
\hline
\end{tabular}

GSE: grape seed extract, VC: vitamin C, VE: vitamin E, MDA: malondialdehyde.

Data as means \pm MSE (mean standard error).

${ }^{*} P<0.05,{ }^{*} P<0.01,{ }^{* * *} P<0.001$; comparisons with positive controls, ${ }^{\mathrm{a}} P<0.01$; comparisons with the same dose of VC; ${ }^{\mathrm{t}} P=0.054$; comparisons with that of VE, (Mann-Whitney $U$ test).

TABLE 2: Effects of GSE, VC, and VE on ulcer indexes and MDA gastric concentrations in rats with ethanol-induced ulcer.

\begin{tabular}{|c|c|c|c|c|c|}
\hline Treatment & Doses $(\mathrm{mg} / \mathrm{kg})$ & Ulcer index (mm) & I $(\%)$ & $\mathrm{MDA}(\mathrm{nmol} / \mathrm{mg}$ of $\mathrm{pt})$ & I $(\%)$ \\
\hline Negative control (vehicle) & 0 & $0 \pm 0^{* * * *}$ & - & $1.02 \pm 0.21^{* *}$ & - \\
\hline Positive control (vehicle + ethanol) & 0 & $72.27 \pm 8.43$ & - & $18.72 \pm 0.76$ & - \\
\hline Vit C + ethanol & 25 & $32.32 \pm 10.1^{*}$ & 55.2 & $10.71 \pm 0.54^{* * *}$ & 45.2 \\
\hline Vit C + ethanol & 250 & $32.67 \pm 8.8^{* *}$ & 54.8 & $7.93 \pm 0.88^{* * *}$ & 61.0 \\
\hline Vit E + ethanol & 25 & $40.94 \pm 9.6^{*}$ & 43.3 & $11.03 \pm 0.57^{* * *}$ & 43.4 \\
\hline Vit E + ethanol & 250 & $29.21 \pm 9.8^{* *}$ & 59.6 & $5.02 \pm 0.59^{* * *}$ & 77.4 \\
\hline GSE + ethanol & 25 & $13.27 \pm 4.8^{* * * a b}$ & 81.6 & $7.84 \pm 0.45^{* * * a a b b}$ & 61.4 \\
\hline GSE + ethanol & 250 & $2.03 \pm 0.69^{* * * a a b}$ & 97.2 & $4.00 \pm 0.28^{* * * a a}$ & 83.2 \\
\hline
\end{tabular}

GSE: grape seed extract, VC: vitamin C, VE: vitamin E, MDA: malondialdehyde.

Data as means \pm MSE (mean standard error).

${ }^{*} P<0.05,{ }^{* *} P<0.01$, ${ }^{* *} P<0.001$; comparisons with positive control, ${ }^{\mathrm{a}} P<0.05$, ${ }^{\text {aa }} P<0.01$ comparisons with the same doses of $\mathrm{VC}$, ${ }^{\mathrm{b}} P<0.05$, bb $P<$ 0.01 comparisons with the same doses of VE, (Mann-Whitney $U$ test).

the development of aspirin-induced gastric ulcers and attenuated the increase of gastric MDA.

Oral administration of VC, VE, and GSE $25 \mathrm{mg} / \mathrm{kg}$ decreased the ulcers $(59.7 \%, 49.3 \%$, and $57.3 \%$, resp.) and gastric MDA $(31.8,52.8$, and $63.6 \%$, resp.) versus the positive controls, while, at $250 \mathrm{mg} / \mathrm{kg}$, they produced ulcer reductions of $69.1 \%, 61.9 \%$, and $56.3 \%$, respectively, and MDA decreases of 67.4, 81.4, and 91.7\%, respectively (comparisons versus positive control rats). The effect of the highest doses of each treatment revealed that the magnitude of ulcer reduction was comparable for all treatments, while MDA decrease with GSE was higher than with VC and tended to be greater, albeit non significantly $(P=0.058)$, than with VE.

Oral administration of ethanol produced gastric ulceration and increased significantly the MDA gastric content (Table 2). All schemes protected against ethanol-induced gastric damage and reduced MDA gastric levels. Treatment with VC, VE, and GSE given at $25 \mathrm{mg} / \mathrm{kg}$ reduced ethanol ulcers $(55.2 \%, 43.3 \%$, and $81.6 \%$, resp.) and attenuated MDA increases $(45.2 \%, 43.4 \%$, and $61.4 \%$, resp.), while it at $250 \mathrm{mg} / \mathrm{kg}$, reduced the ulcers by $54.8 \%, 59.6 \%$, and $97.2 \%$, respectively, and decreased MDA by $61.0 \%, 77.4 \%$, and $83.2 \%$, respectively, versus the positive controls.
Oral treatment with GSE reduced ethanol-induced ulcers more effectively than VC and VE, while it produced reductions of MDA levels greater than VC, but similar to VE. The lowest dose of GSE, however, lowered MDA concentrations more than the same dose of VE.

Overall, GSE 25 and $250 \mathrm{mg} / \mathrm{kg}$ produced greater percent reductions of ethanol than aspirin-induced ulcers. The reductions of gastric MDA levels with the lowest dose $(25 \mathrm{mg} / \mathrm{kg})$ of GSE were similar in both models, while the MDA reduction with the highest dose $(250 \mathrm{mg} / \mathrm{kg})$ seems to be greater in rats with aspirin-induced ulceration.

The experimental models here used share similarities and differences. Increased oxidative stress and ROS production are pathogenic mechanisms of both models [49, 12]. Nevertheless, the key mechanism of NSAID-induced gastric ulceration results from the irreversible and nonselective inhibition of COX activity, which interferes with the synthesis from PG, triggering the effects derived of PG depletion, and shuttles the arachidonic acid metabolism towards the lipoxygenase pathway, increasing the formation of vasoconstrictor leukotrienes (LTs) $[6,7,10,11]$.

The gastric ulceration induced by oral administration of ethanol to rats involves other mechanisms in addition to the increase of oxidative stress and ROS formation. So, ethanol 
also depletes PG concentration due to its necrotizing action on the gastric mucosa and, thus, shares the consequences of PG as oral administration of aspirin does, as increased vascular permeability and decreased gastric mucosa microcirculation. Also, the necrotizing action of ethanol decrease, gastric mucus secretion and impairs the quality of mucus composition [42].

The protective effect of VC involves the reduction of lipid peroxidation in the gastric mucosa, which preserves the gastric microcirculation. VC stimulates the expression of the antioxidant and vasodilator heme oxygenase enzyme in the gastric epithelium and inhibits the expression of inducible nitric oxide synthase enzyme $[9,43,44]$. It makes sense, therefore, that VC had been effective to protecting against both aspirin- and ethanol-induced ulcers. The highest dose of $\mathrm{VC}$ reduced aspirin-induced ulcer more markedly (about $70 \%$ ) than ethanol ulcers (about 55\%), while the effects on gastric MDA (67\% and 61\%, resp.) were comparable. These results suggest that although the effects of VC against aspirin and ethanol ulcers may be attributable to the reduction of lipid peroxidation, this is not the only mechanism involved in the gastroprotective effect on aspirin-induced ulcers. Further studies must dilucidate the direct or indirect mechanism of action that supports the gastroprotective and antioxidant effects of Vitamin C.

Oral administered VE has been shown to be effective against ethanol and NSAIDs-induced ulcers, an action that involves the reduction of lipid peroxidation and the increases of the activity of endogenous antioxidant enzymes, like superoxide dismutase, catalase, and glutathione peroxidise [25-27]. Then, the fact that VE was effective in the two models here used was expected. The highest dose of VE produced similar reductions (about 60\%) of aspirin- and ethanolinduced ulcers and of MDA values (roughly about $80 \%$ ). Then, although these results suggest that the gastroprotective effects of VE may be related to its antioxidant effects, these last ones seem to be greater, a finding without conclusive explanation.

The effect of VC for protecting against aspirin-induced ulcers ( $\cong 70 \%$ of inhibition) was apparently, not significantly, greater than that of $\mathrm{VE}(\cong 62 \%$ of inhibition), while the effects of both treatments on ethanol ulcers were grossly comparable. This result, however, is slightly different from that reported in the model of water-restrain-stress- (WRS) induced ulcers, in which VE was more effective than VC, despite the fact that both substances exerted their protective actions through antioxidant and anti-inflammatory effects [45].

Oral administration of GSE protected against ethanolinduced gastric ulcers in rats more effectively than VE and VC, wherein the lowest dose of GSE produced a reduction of these ulcers (about $82 \%$ ) and the highest dose practically abolished (about 97\% reduction) the ethanolinduced ulcers. Although the decreases of MDA levels with GSE $(\cong 83 \%)$ were greater than with VC $(\cong 61 \%)$ or VE ( $\cong 77 \%$ ), it should be noted that ethanol ulcer reduction with GSE was greater than MDA decreases, consistent with the fact that the protection of GSE against ethanol-induced ulcers depends not only on its scavenging properties, but on its ability for lowering increase MPO and consequent neutrophil infiltration $[25,46]$ as well.

The magnitude of aspirin-induced ulcer reduction, comparable for all treatments, was apparently greater with VC $250 \mathrm{mg} / \mathrm{kg}$ (about 70\%) than with the same dose of GSE (about 57\%). By contrast, the same dose of GSE decreased MDA (about 97\%) more markedly than VC (about 83.2\%). These results suggest that although the efficacy of GSE on this model should be attributable to its ability to lower lipid peroxidation, it did not reduce other mechanisms leading to aspirin-induced ulcers.

\section{Conclusions}

GSE prevented ethanol-induced gastric ulcers more effectively than VC or VE, while its protection against aspirin ulcers was comparable for all treatments. GSE produced the greatest reductions of gastric MDA in both models. Then, the gastroprotective effects of GSE may be related, at least partially, to its ability for reducing lipid peroxidation in the gastric mucosa. Further studies must compare the therapeutic effects of GSE, VC, and VE on ethanol- and aspirin-induced gastric ulcer in rats.

\section{References}

[1] K. Ramakrishnan and R. C. Salinas, "Peptic ulcer disease," American Family Physician, vol. 76, no. 7, pp. 1005-1012, 2007.

[2] C. Tasman-Jones, "Pathogenesis of peptic ulcer disease and gastritis: importance of aggressive and cytoprotective factors," Scandinavian Journal of Gastroenterology, vol. 21, supplement 22, pp. 1-5, 1986.

[3] L. Laine, K. Takeuchi, and A. Tarnawski, "Gastric mucosal defense and cytoprotection: bench to bedside," Gastroenterology, vol. 135, no. 1, pp. 41-60, 2008.

[4] J. Kountouras, D. Chatzopoulos, and C. Zavos, "Reactive oxygen metabolites and upper gastrointestinal diseases," HepatoGastroenterology, vol. 48, no. 39, pp. 743-751, 2001.

[5] S. Demir, M. Yilmaz, M. Köseoğlu, N. Akalin, D. Aslan, and A. Aydin, "Role of free radicals in peptic ulcer and gastritis," Turkish Journal of Gastroenterology, vol. 14, no. 1, pp. 39-43, 2003.

[6] B. J. Whittle, "Gastrointestinal effects of nonsteroidal antiinflammatory drugs," Fundamental and Clinical Pharmacology, vol. 17, no. 3, pp. 301-313, 2003.

[7] D. Lamarque, "Pathogenesis of gastroduodenal lesions induced by non-steroidal anti-inflammatory drugs," Gastroenterologie Clinique et Biologique, vol. 28, pp. C18-C26, 2004.

[8] G. Sener-Muratoğlu, K. Paskaloğlu, S. Arbak et al., "Protective effect of famotidine, omeprazole, and melatonin against acetylsalicylic acid-induced gastric damage in rats," Digestive Diseases and Sciences, vol. 46, no. 2, pp. 318-330, 2001.

[9] T. Pohle, T. Brzozowski, J. C. Becker et al., "Role of reactive oxygen metabolites in aspirin-induced gastric damage in humans: gastroprotection by vitamin C," Alimentary Pharmacology and Therapeutics, vol. 15, no. 5, pp. 677-687, 2001.

[10] M. Jainu and S. Devi, "Attenuation of neutrophil infiltration and proinflammatory cytokines by Cissus quadrangularis: a possible prevention against gastric ulcerogenesis," Journal of Herbal Pharmacotherapy, vol. 5, no. 3, pp. 33-42, 2005. 
[11] M. Jainu, V. Mohan, and S. Devi, "Protective effect of Cissus quadrangularis on neutrophil mediated tissue injury induced by aspirin in rats," Journal of Ethnopharmacology, vol. 104, no. 3, pp. 302-305, 2006.

[12] R. Hernandez-Munoz, C. Montiel-Ruiz, and O. VazquezMartinez, "Gastric mucosal cell proliferation in ethanolinduced chronic mucosal injury is related to oxidative stress and lipid peroxidation in rats," Laboratory Investigation, vol. 80, supplement 8, pp. 1161-1169, 2000.

[13] H. Araki, H. Ukawa, Y. Sugawa, K. Yagi, K. Suzuki, and K. Takeuchi, "The roles of prostaglandin E receptor subtypes in the cytoprotective action of prostaglandin E2 in rat stomach," Alimentary Pharmacology \& Therapeutics, vol. 14, supplement 1, pp. 116-124, 2000.

[14] A. Slomiany, M. Morita, S. Sano, J. Piotrowski, D. Skrodzka, and B. L. Slomiany, "Effect of ethanol on gastric mucus glycoprotein synthesis, translocation, transport, glycosylation, and secretion," Alcoholism: Clinical and Experimental Research, vol. 21, no. 3, pp. 417-423, 1997.

[15] S. Sánchez-Fidalgo, I. Martín-Lacave, M. Illanes, and V. Motilva, "Angiogenesis, cell proliferation and apoptosis in gastric ulcer healing. Effect of a selective cox-2 inhibitor," European Journal of Pharmacology, vol. 505, no. 1-3, pp. 187194, 2004.

[16] R. Amanvermez, O. K. Tunçel, S. Demir, M. Kefeli, Y. Bek, and C. Celik, "Protective effects of cysteine, methionine and vitamin C on the stomach in chronically alcohol treated rats," Journal of Applied Toxicology, vol. 28, no. 5, pp. 591-598, 2008.

[17] R. E. Beyer, "The role of ascorbate in antioxidant protection of biomembranes: interaction with vitamin E and coenzyme Q," Journal of Bioenergetics and Biomembranes, vol. 26, no. 4, pp. 349-358, 1994.

[18] E. Jonas, A. Dwenger, and A. Hager, "In vitro effect of ascorbic acid on neutrophil-endothelial cell interaction," Journal of Bioluminescence and Chemiluminescence, vol. 8, no. 1, pp. 1520, 1993.

[19] J. C. Becker, N. Grosser, P. Boknik, H. Schröder, W. Domschke, and T. Pohle, "Gastroprotection by vitamin C-a heme oxygenase-1-dependent mechanism?” Biochemical and Biophysical Research Communications, vol. 312, no. 2, pp. 507512, 2003.

[20] P. C. Konturek, J. Kania, E. G. Hahn, and J. W. Konturek, "Ascorbic acid attenuates aspirin-induced gastric damage: role of inducible nitric oxide synthase," Journal of Physiology and Pharmacology, vol. 57, supplement 5, pp. 125-136, 2006.

[21] D. C. Liebler, "Antioxidant chemistry of alpha-tocopherol in biological systems. Roles of redox cycles and metabolism," Subcellular Biochemistry, vol. 30, pp. 301-317, 1998.

[22] N. Yoshida, T. Yoshikawa, H. Manabe et al., "Vitamin E protects against polymorphonuclear leukocyte-dependent adhesion to endothelial cells," Journal of Leukocyte Biology, vol. 65, no. 6, pp. 757-763, 1999.

[23] D. Rocksén, B. Ekstrand-Hammarström, L. Johansson, and A. Bucht, "Vitamin E reduces transendothelial migration of neutrophils and prevents lung injury in endotoxin-induced airway inflammation," American Journal of Respiratory Cell and Molecular Biology, vol. 28, no. 2, pp. 199-207, 2003.

[24] K. Jaarin, M. Renuvathani, M. I. Nafeeza, and M. T. Gapor, "Effect of palm vitamin E on the healing of ethanol-induced gastric injury in rats," International Journal of Food Sciences and Nutrition, vol. 51, pp. S31-S41, 2000.

[25] K. Jaarin, M. T. Gapor, M. I. Nafeeza, and A. M. Fauzee, "Effect of various doses of palm vitamin $\mathrm{E}$ and tocopherol on aspirin-induced gastric lesions in rats," International Journal of Experimental Pathology, vol. 83, no. 6, pp. 295-302, 2002.

[26] M. Fesharaki, A. Nasimi, S. Mokhtari, R. Mokhtari, R. Moradian, and N. Amirpoor, "Reactive oxygen metabolites and anti-oxidative defenses in aspirin-induced gastric damage in rats: gastroprotection by Vitamin E," Pathophysiology, vol. 13, no. 4, pp. 237-243, 2006.

[27] M. Balu, P. Sangeetha, G. Murali, and C. Panneerselvam, "Agerelated oxidative protein damages in central nervous system of rats: modulatory role of grape seed extract," International Journal of Developmental Neuroscience, vol. 23, no. 6, pp. 501507, 2005.

[28] M. Balu, P. Sangeetha, D. Haripriya, and C. Panneerselvam, "Rejuvenation of antioxidant system in central nervous system of aged rats by grape seed extract," Neuroscience Letters, vol. 383, no. 3, pp. 295-300, 2005.

[29] A. Devi, A. B. Jolitha, and N. Ishii, "Grape seed proanthocyanidin extract (GSPE) and antioxidant defense in the brain of adult rats," Medical Science Monitor, vol. 12, no. 4, pp. 124$129,2006$.

[30] J. Busserolles, E. Gueux, B. Balasińska et al., "In vivo antioxidant activity of procyanidin-rich extracts from grape seed and pine (Pinus maritima) bark in rats," International Journal for Vitamin and Nutrition Research, vol. 76, no. 1, pp. 22-27, 2006.

[31] I. Rodríguez, J. Illnait, V. Molina et al., "Comparison of the antioxidant effects of D-002 (beeswax alcohols) and grape seed extract (GSE) on plasma oxidative variables in healthy subjects," Latin American Journal of Pharmacy, vol. 29, pp. 255-562, 2010.

[32] P. Kar, D. Laight, H. K. Rooprai, K. M. Shaw, and M. Cummings, "Effects of grape seed extract in Type 2 diabetic subjects at high cardiovascular risk: a double blind randomized placebo controlled trial examining metabolic markers, vascular tone, inflammation, oxidative stress and insulin sensitivity," Diabetic Medicine, vol. 26, no. 5, pp. 526-531, 2009.

[33] M. Saito, H. Hosoyama, T. Ariga, S. Kataoka, and N. Yamaji, "Antiulcer activity of grape seed extract and procyanidins," Journal of Agricultural and Food Chemistry, vol. 46, no. 4, pp. 1460-1464, 1998.

[34] H. Zengil, E. Onuk, Z. S. Ercan, and R. K. Turker, "Protective effect of iloprost and UK 38485 against gastric mucosal damage induced by various stimuli," Prostaglandins Leukotrienes and Medicine, vol. 30, no. 2-3, pp. 61-67, 1987.

[35] A. Ohara, S. Sugiyama, H. Hoshino et al., "Reduction of adverse effects of indometacin by anti-allergic drugs in rat stomachs," Arzneimittel-Forschung/Drug Research, vol. 42, no. 9, pp. 1115-1118, 1992.

[36] H. Ohkawa, N. Ohishi, and K. Yagi, "Assay for lipid peroxides in animal tissues by thiobarbituric acid reaction," Analytical Biochemistry, vol. 95, no. 2, pp. 351-358, 1979.

[37] P. A. Kumar and G. Rajagopal, "Lipid peroxidation in erythrocytes of patients with type 2 diabetes mellitus," Indian Journal of Clinical Biochemistry, vol. 18, no. 1, pp. 71-74, 2003.

[38] R. Premanand, P. H. S. Kumar, and A. Mohan, "Study of thiobarbituric reactive substances and total reduced glutathione as indices of oxidative stress in chronic smokers with and without chronic obstructive pulmonary disease," The Indian Journal of Chest Diseases and Allied Sciences, vol. 49, no. 1, pp. 9-12, 2007.

[39] S. V. Tembhurne and D. M. Sakarkar, "Protective effect of Murraya koenigii (L) leaves extract in spreptozotocin induced diabetics rats involving possible antioxidant mechanism," Journal of Medicinal Plants Research, vol. 4, pp. 2418-2423, 2010. 
[40] T. Matsunami, Y. Sato, T. Sato, and M. Yukawa, "Antioxidant status and lipid peroxidation in diabetic rats under hyperbaric oxygen exposure," Physiological Research, vol. 59, no. 1, pp. 97$104,2010$.

[41] M. A. Marxwell, S. M. Haas, L. L. Beiber, and N. E. Tolbert, "A modification of the Lowry procedure to simplify protein determination in membrane lipoprotein samples," Analytical Biochemistry, vol. 87, pp. 206-209, 1987.

[42] M. G. Repetto and S. F. Llesuy, "Antioxidant properties of natural compounds used in popular medicine for gastric ulcers," Brazilian Journal of Medical and Biological Research, vol. 35, no. 5, pp. 523-534, 2002.

[43] T. Brzozowski, S. KwiecieÅ, P. Konturek et al., "Comparison of nitric oxide-releasing NSAID and vitamin $\mathrm{C}$ with classic NSAID in healing of chronic gastric ulcers; involvement of reactive oxygen species," Medical Science Monitor, vol. 7, no. 4, pp. 592-599, 2001.

[44] J. C. Becker, N. Grosser, P. Boknik, H. Schröder, W. Domschke, and T. Pohle, "Gastroprotection by vitamin C-a heme oxygenase-1-dependent mechanism?” Biochemical and Biophysical Research Communications, vol. 312, no. 2, pp. 507512, 2003.

[45] Y. Ohta, Y. Imai, S. Kaida, Y. Kamiya, M. Kawanishi, and I. Hirata, "Vitamin E protects against stress-induced gastric mucosal lesions in rats more effectively than vitamin C," BioFactors, vol. 36, no. 1, pp. 60-69, 2010.

[46] G. Z. Wang, G. P. Huang, G. L. Yin et al., "Aspirin can elicit the recurrence of gastric ulcer induced with acetic acid in rats," Cellular Physiology and Biochemistry, vol. 20, no. 1-4, pp. 205212, 2007. 

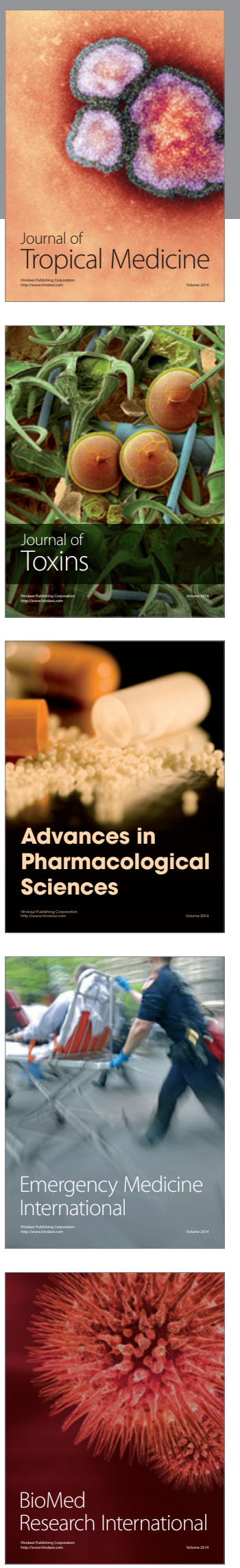
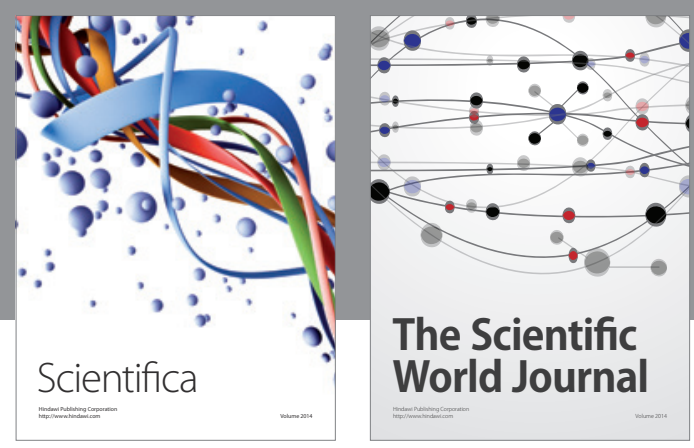

The Scientific World Journal
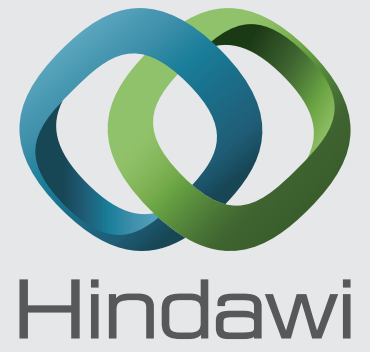

Submit your manuscripts at

http://www.hindawi.com
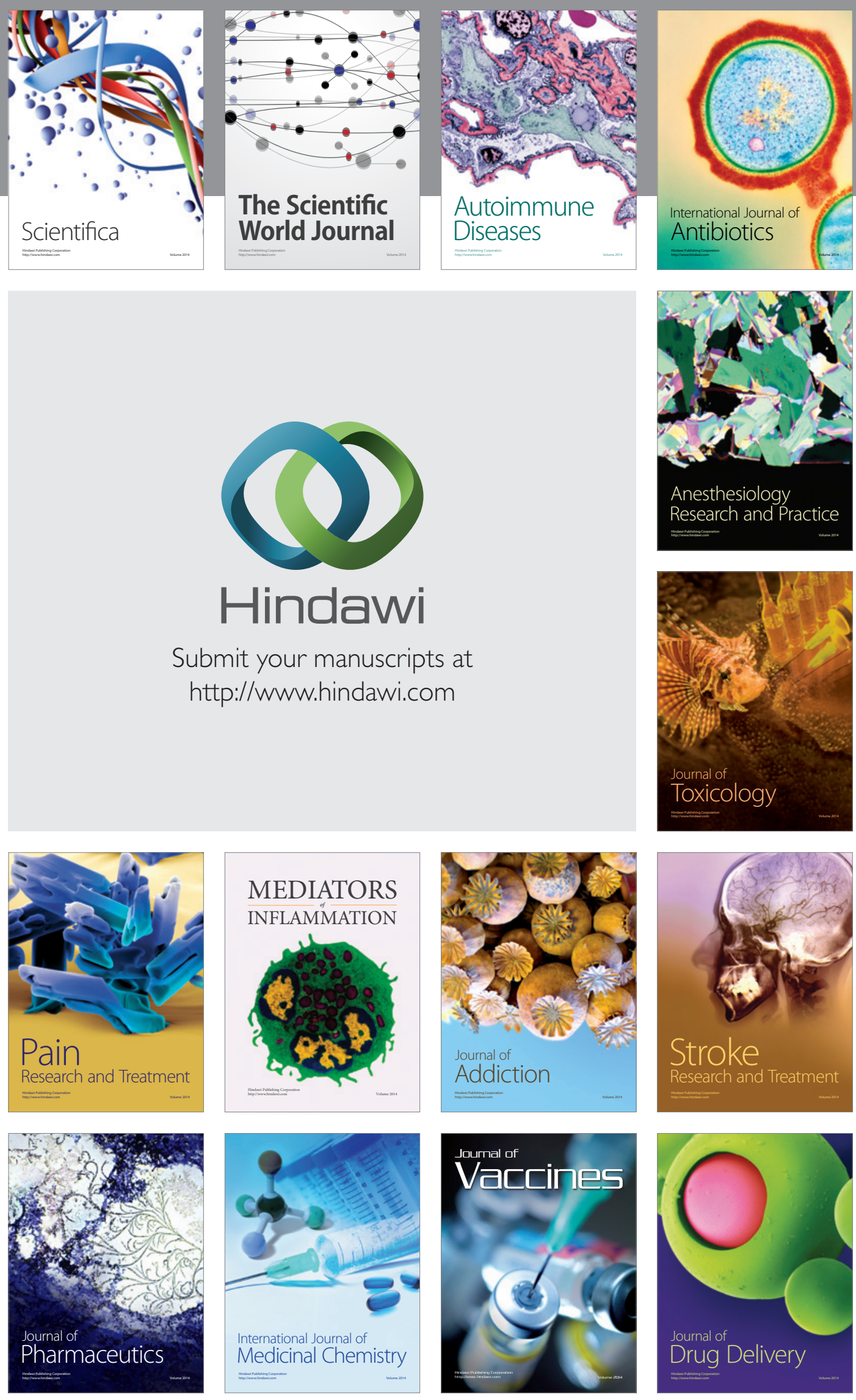